\title{
The Potential Application of Risk Assessment to the Breakthrough Propulsion Physics Project
}

\author{
Edward J. Zampino $\bullet$ NASA Glenn Research Center $\bullet$ Cleveland \\ Marc G. Millis• NASA Glenn Research Center • Cleveland
}

Key Words: Success Event, Success Tree, Success Tree Analysis, Defect Detection and Prevention, Project Success Analysis, Emerging Science, Ultra-Reliability, Ultra-Maintainability, Hypothetical-system Availability Analysis

\section{SUMMARY \& CONCLUSIONS}

In May of 2001, researchers at the NASA Glenn Research center in Cleveland Ohio began exploring the possibility of applying Risk Assessment to a frontier propulsion project called Breakthrough Propulsion Physics (BPP). The goals of BPP are the drastic reduction or elimination of propellant mass, the attainment of hyper-fast space travel (approaching or exceeding) the speed of light, and new methods for onboard energy conversion. The BPP challenges transcend mere engineering and forces a re-examination of the fundamental physics from which technology is developed. Presently, BPP is at a maturity level of emerging science. The challenge is formidable: to apply risk assessment to a project that will not have conceptual designs for a long time to come. However, we suggest that it still makes a great deal of sense to think about the risks associated with various paths of research. In particular, it is suggested that there are a number of potential areas where Risk Assessment can be applied even when a project is at the maturity level of emerging science. It is believed that with the concepts and tools of Risk Assessment, more is possible than assessing potential reliability and maintainability. There is more to the story than the obvious need for greater Reliability. We are proposing that Risk Assessment Tools can be applied to guide research success.

\section{INTRODUCTION}

Interstellar space travel will pose many difficult scientific problems. Here, we will discuss only a few. One of the most formidable problems is the Propellant Mass Problem. Chemical propellant mass requirements for interstellar travel are nothing less than "astronomical." In order to send a vehicle using chemical propellant with the mass of a space shuttle to our nearest star at the leisurely pace of a 1000 year trip time would require $10^{119} \mathrm{~kg}$. of propellant.[1] It may seem that the solution to the Propellant Mass Problem is just a matter of developing more efficient rockets. Unfortunately, exhaust cannot leave a rocket with a velocity that exceeds the velocity of light in a vacuum. This upper limit to the speed of material objects in the universe imposed by Relativistic Dynamics places a serious constraint on how efficient a rocket can be. A perfect rocket would have a propellant of matter and anti-matter that react in a controlled way producing a photon exhaust. A fuel of anti-matter and ordinary matter to react with it would have the smallest ratio of stored energy $\mathrm{E}$, to total mass $\mathrm{M}$, that is physically possible, namely $\mathrm{E} / \mathrm{M}=\mathrm{c}^{2}$. A typical interstellar mission will require at least four phases for a round trip: two phases where acceleration is required and two phases where deacceleration is required. Calculations using the Rocket Equation of Relativistic Dynamics show that the amount of anti-matter required for an interstellar mission with a perfect rocket will be very difficult to produce. [2]

Thus, the challenge is to explore the possibility that propulsion may be achieved without propellant. This approach requires that space must have some kind of dynamic structure that can be reacted against in order to satisfy the Law of Conservation of Momentum. Examples of field-drive techniques might be the application of exotic or specially conditioned electromagnetic fields to create antigravity or to "shut off" inertia. A second challenge is to explore the possibility of hyper-fast space travel to exceed the speed of light. Physicists are studying loop holes in Einstein's General Theory of Relativity concerning spacetime "warp-drive" bubbles and induced wormholes. [3] Experiments in hyper-fast travel involve the quantum mechanical tunneling of light through a potential barrier with an apparent super-luminal speed. Hyper-fast travel would eliminate the enormous round-trip times required for missions to the distant stars at "sub-light" speeds. Thirdly, there has to be a method for abundant on-board energy conversion. Tremendous power will be needed to power such star ships of the future. Physicists are therefore studying the possible extraction of power from the zeropoint vacuum fluctuations of the electromagnetic field. [4] However, there is the fourth great problem of interstellar space travel that will exist for any technology: the reliability and maintainability problem.

At interstellar distances, the distress calls carried by electromagnetic waves will take years to reach earth. There will be no re-supply missions to provide spares. Vehicle reliability must be extremely high and must transcend the reliability of any current technology. Maintainability must be ultra-advanced and able to detect, isolate, and correct for even the slightest onset of anomalous conditions.

It is not difficult to see that Risk Assessment will have a direct application to the Interstellar propulsion problem. But more is possible than merely assessing the potential reliability and maintainability of the ultimate propulsion technology. What if Risk Assessment tools could be applied 
even in the research process? The authors believe that this is possible. Ideas are presented here to show how this may be approached.

\subsection{GLOSSARY}

- Success Event: A specific event occurring as part of a research path or system operation that represents a successful outcome according to some objective criteria.

- Success Tree Analysis: The logical complement of a fault tree analysis. The Top-Level Event represents the desired successful outcome for a project or system. The tree defines the logical and causal relationship between events that bring about the Top-Level success event. The analysis of this tree provides cut-sets for success and importance measures relating to the basic events that enable the TopLevel success event. [5]

- Traceability Tree: A project diagram that depicts the relationships between various research approaches and their subject matter categories.

- Emerging Science: An intermediate stage between knowledgeable conjecture based on limited scientific knowledge and Science. (when you know what is theoretically possible)

- Expert Elicitation: The process of interviewing experts in any field of endeavor in order to obtain estimates of quantitative parameters and critical insights based on expert knowledge and understanding.

- Defect Detection and Prevention (DDP): A methodology to determine the residual risk after applying process controls and balancing risk reduction with resources. It considers mission requirements, the impact of failure modes on attainment of requirements, a priori probabilities of occurrence, and measures for screening out project failure modes. ( Preventative Measures, Analysis, Process Controls, Testing) [6]

- Project Success Analysis: The logical complement of DDP. A proposed methodology to determine research value and value improvement for a project. Considers Science objectives, Success paths, a priori probabilities for success, and flaws or weaknesses that threaten success.

- Hypothetical System Availability Studies: The practice of developing an availability model (block diagram) for a hypothetical (or proposed) system design, in order to estimate system operational availability and sensitivities in the design.

- Ultra-Reliability: Extremely high reliability needed to support Interstellar missions where there is nearly a zero tolerance for critical system failures due to the immense distances away from earth.

- Ultra-Maintainability: The ability to detect, isolate, and correct for even the onset of anomalous conditions that would be encountered on an interstellar flight.

\subsection{ACRONYMS}

The following are acronyms:

BPP- Breakthrough Propulsion Physics

DDP- Defect Detection and Prevention

FMEA - Failure Modes and Effects Analysis

FTA - Fault Tree Analysis

GRC - NASA Glenn Research Center

MLDT- Mean-logistics delay time

MRT- Mean Repair time

MTBDE Mean-time-between downing events

MTBF- Mean-time-between failures

MTTR- Mean-time-to-repair

PRA - Probabilistic Risk Assessment

PSA - Project Success Analysis

RAM- Reliability, Availability, and Maintainability

R\&M- Reliability and Maintainability

STA - Success Tree Analysis

ZPF- Zero-point fluctuations of the vacuum electromagnetic field predicted by Quantum Mechanics.

Figure 1: A Graphical Depiction of Possible Flaws, Weaknesses, and Difficulties

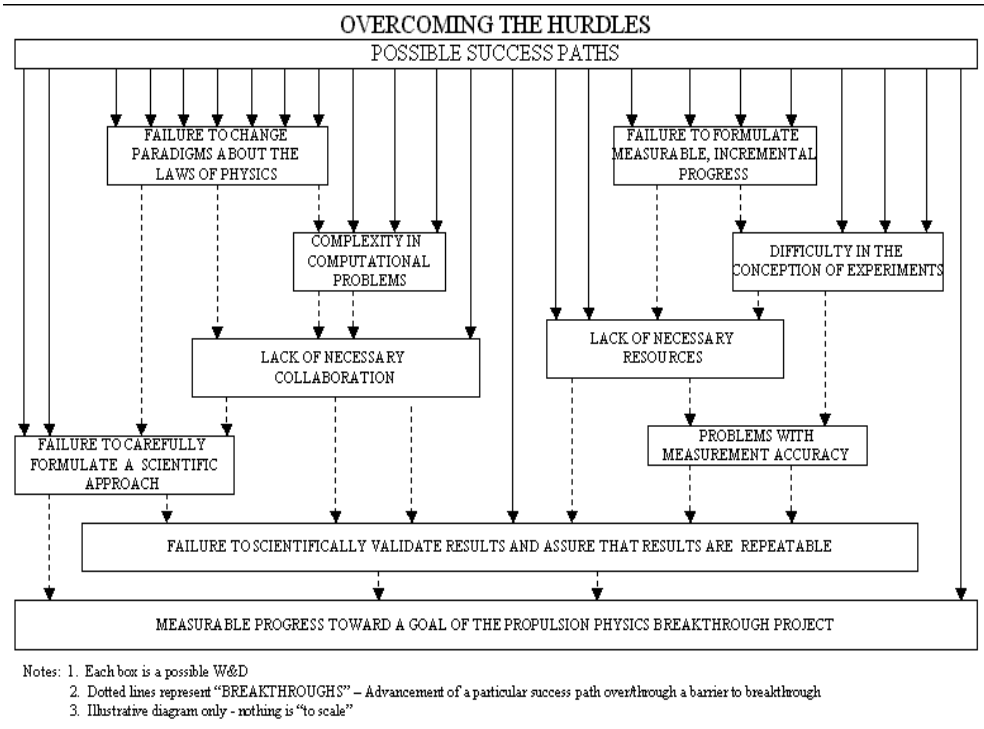




\section{DIFFERENCES IN PERSPECTIVE}

One of the first things to note is the difference in perspective about risk between the Reliability engineer and the Research physicist. The Reliability engineer worries about failures, faults, failure mode propagation , probability of system failure and the risk of having catastrophic events. On the other hand, the Research physicists worry about success events, finding success paths, probability of success, the risk of overlooking a scientific discovery, and balancing resources and benefits. The more productive approach with the research activity, at the maturity level of emerging science, was to express risk in positive terms rather than negative. (Benefit replaces severity of failure and Probability of Success replaces Probability of Failure) When the probability of Success is found, we can always find the Probability of Failure by simply subtracting the probability of success from one. The success oriented approach to risk appears to be more acceptable to the research process. However, when considering the analysis of system concepts that are hypothetical or research infrastructure one can always revert back to the usual risk assessment approach where we focus on the probability of failure and severity of failure events.

\section{THE OVERALL APPROACH}

The Research Project goal is to produce incremental, credible, and measurable progress. The idea behind breakthrough is that there is some point of investment where a revolutionary idea, method, or perception will drastically boost project performance toward the attainment of the scientific goal. This is in contrast to the continued effort to attain the goal using conventional methods that may be asymptotic to a performance limit with increasing investments. The idea is to combine physics, engineering, technology, and risk assessment to achieve the total breakthrough. Several ideas for the risk assessment activity are suggested as a way to accomplish this.

1.The application of Project Success Analysis to understand Project Risks

2.Success Tree Analysis

3.Preliminary RAM trade studies to compare advanced propulsion concepts for a typical interstellar mission

4.FMEA and/or Fault Tree Analysis of experiment designs 5.Physics of Failure studies applied to hypothetical devices

6.Software Product Assurance applied to advanced codes

7.Analysis of Human Error possibilities

\section{THREE EXAMPLES OF POTENTIAL APPLICATIONS}

The three examples that we wish to discuss here are:

1. Can Defect Detection and Prevention (DDP) be translated into a Project Success Analysis (PSA)?

2. Can an Operational Availability Analysis of a hypothetical field drive propulsion vehicle for an Interstellar Mission be performed?
3. Can we develop a Success Tree Analysis of Scientific Research Approaches?

Defect Detection and Prevention (DDP) is a methodology developed by Dr. Steven Cornford at NASA JPL to determine the residual risk and risk balancing profiles for a project. It starts by compiling Mission requirements and proceeds to evaluate the impact of Project failure modes on the attainment of requirements. ( Example: What percentage of the requirement is lost if the failure mode occurs?) In DDP, failure modes are taken in the most general sense: they are literally anything that can go wrong. Then, apriori probabilities of occurrence for failure modes are considered. These apriori probabilities mean: What is the probability that a failure mode will occur given that we do nothing to prevent it? The second part of DDP is to consider the measures that are taken to "screen out" failure modes from the Project. These measures or actions are defined as: Preventative measures, Analysis, Controls on Processes, and Testing (PACTs).

In searching for a way to apply DDP to the Breakthrough Propulsion Physics Project, the authors conceived of the idea to translate DDP into its logical complement methodology which can be called Project Success Analysis (PSA). It is believed that PSA should be adaptable to the research process and is an alternative way to look at Risk. Below, in figures 2 and 3 two charts compare the DDP methodology and PSA side by side.

The PSA would focus on the paths to success for attaining Science objectives. ( Possible approaches to attain the science goal as opposed to failure modes) An apriori probability for success along each path would be estimated: What is the probability that a success path will be completed given ideal conditions? The ideal conditions would pertain to very minimal human error and minimal project impediments and difficulties. The Fraction of science objectives achieved (gained) by completing each success path would be estimated. [ See Note 1] (As opposed to the loss of objectives due to the occurrence of a failure mode) Estimates of the apriori probability and the fraction of science objectives attained would be made by expert elicitation. (See Note 2)

The project flaws, weaknesses, and difficulties (W\&Ds) that threaten success would be determined. ( A graphical depiction is shown in figure 1. ) The impact on probability of success for various success paths considering the weaknesses and difficulties is estimated. Analogous to DDP, instead of a risk histogram, a value histogram is developed which will essentially express the probability of success times the benefit of attaining the goal. This would just be a positive way of assessing risk in terms of positively oriented parameters that are preferred by the researchers. However, the analysis will force the research team to consider the real threats to success, the probabilities that they assign to various approaches for the research objective, how these probabilities are affected by the inherent weaknesses in the project (what the reliability engineer would call the possible failure modes) and what types of actions can be taken to eliminate or avoid potential problems. 
Figure 2: Defect Detection and Prevention compared with Project Success Analysis

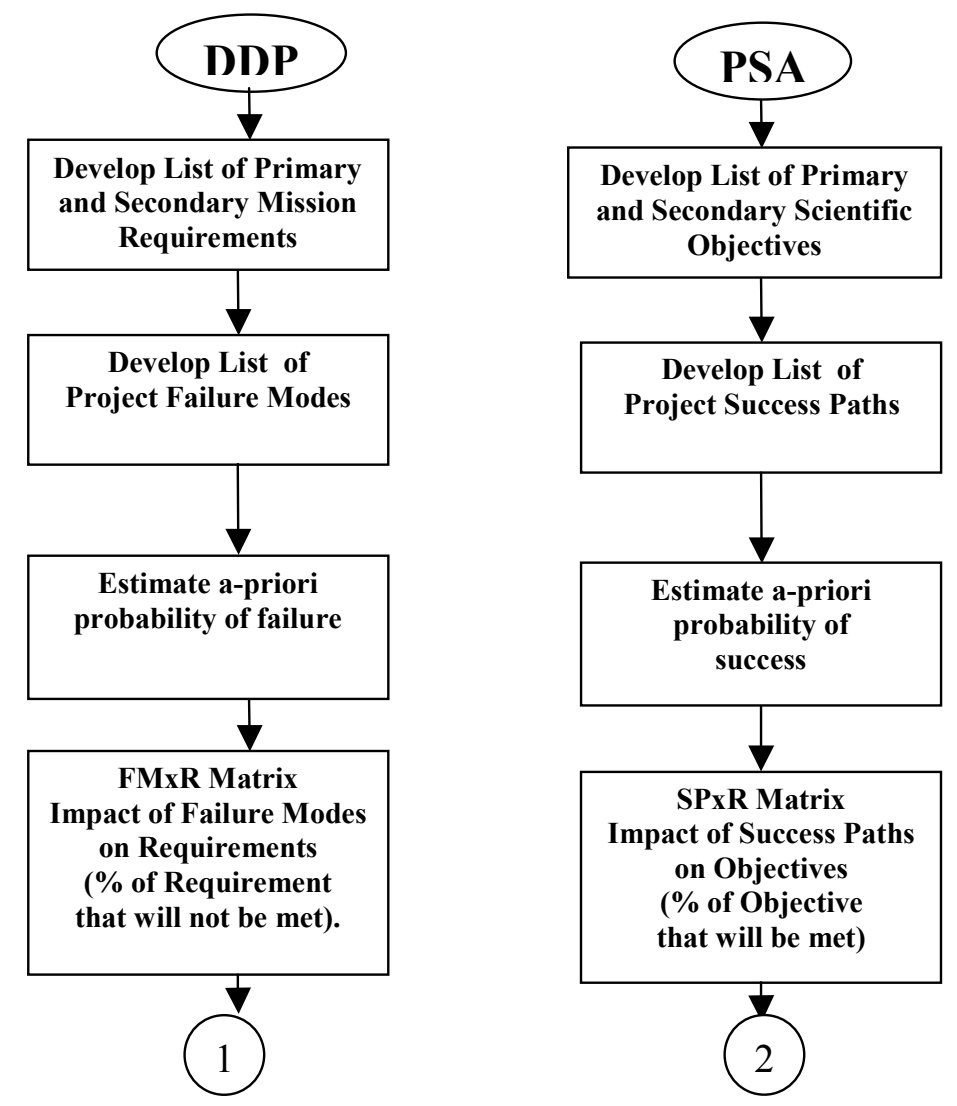

Figure 3: Defect Detection and Prevention compared with Project Success Analysis ( Continued )

\begin{tabular}{|c|}
\hline $\begin{array}{l}\text { Determine PACTs } \\
\text { Measures to be taken To } \\
\text { screen out Failure } \\
\text { Modes }\end{array}$ \\
\hline 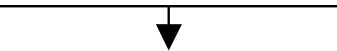 \\
\hline $\begin{array}{l}\text { PACTs vs. FM Matrix } \\
\text { Effectiveness } \\
\text { of PACTs }\end{array}$ \\
\hline $\begin{array}{c}\text { Risk Histogram } \\
\text { (Residual Risk) } \\
\text { Severity x Probability }\end{array}$ \\
\hline$\frac{1}{7}$ \\
\hline $\begin{array}{l}\text { Risk Trade Off : Remove } \\
\text { \& Add PACTs. Accept } \\
\text { more Risk on some } \\
\text { items to Reduce Risk on } \\
\text { others }\end{array}$ \\
\hline 7 \\
\hline $\begin{array}{l}\text { Attain Balanced } \\
\text { Risk Profile }\end{array}$ \\
\hline
\end{tabular}

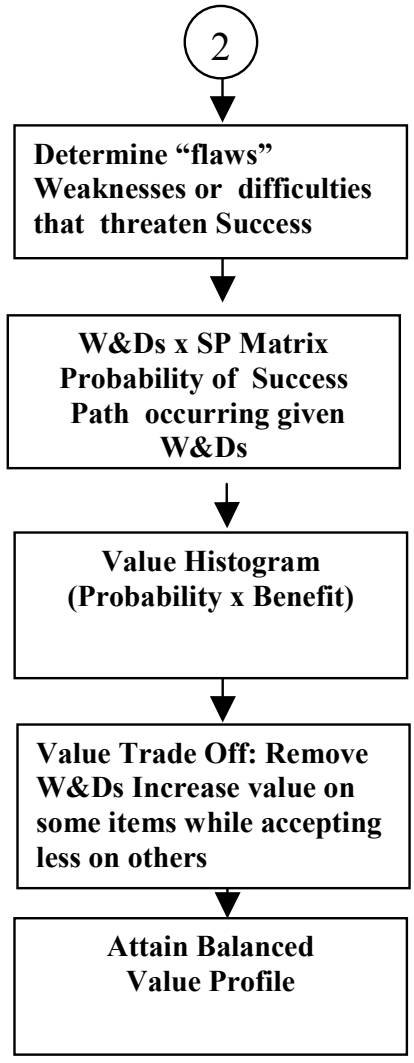


Another proposed application to the BPP project would be the operational availability analysis of a hypothetical fielddrive propulsion vehicle. There are a number of software tools that can be used to construct these models and perform failure simulations over projected interstellar mission times. An example might be to construct an availability model for a hypothetical system using RAPTOR. (Rapid Availability Prototyping for Test and Operational Readiness) currently owned and developed by ARINC Corporation.

This approach might be to construct a block diagram assuming basic types of sub-systems to carry out functions such as: life support, power storage and management, command and data management, communications, fielddrive propulsion, navigation, and shielding.

As a starting point, very high levels of active or standby redundancy could be assumed in the model. Very high MTBF, and low MTTR could be assumed for each redundant unit. It can be assumed that for an interstellar mission, a small set of priority spares are stowed on-board. With each spare is associated a low MLDT because of the proximity of spares to the actual repair or replacement sites on the vehicle. We can gradually reduce the design redundancy, unit MTBF, on-board sparing, and incrementally increase MTTR to reflect hypothetical reductions in reliability and maintenance efficiency. RAM parameters can be changed either individually or in combination. System failure simulations can be run to calculate the resulting operational availability, MTBDE, MRT, and reliability without repair. Thus, in these sensitivity studies, we can search for some optimal system architecture and $\mathrm{R} \& \mathrm{M}$ allocations that will support the mission availability requirement. An example of such a model is shown below: a screen shot from RAPTOR.

Figure 4: Screen shot from RAPTOR Plus showing an availability model for a Hypothetical Field drive system

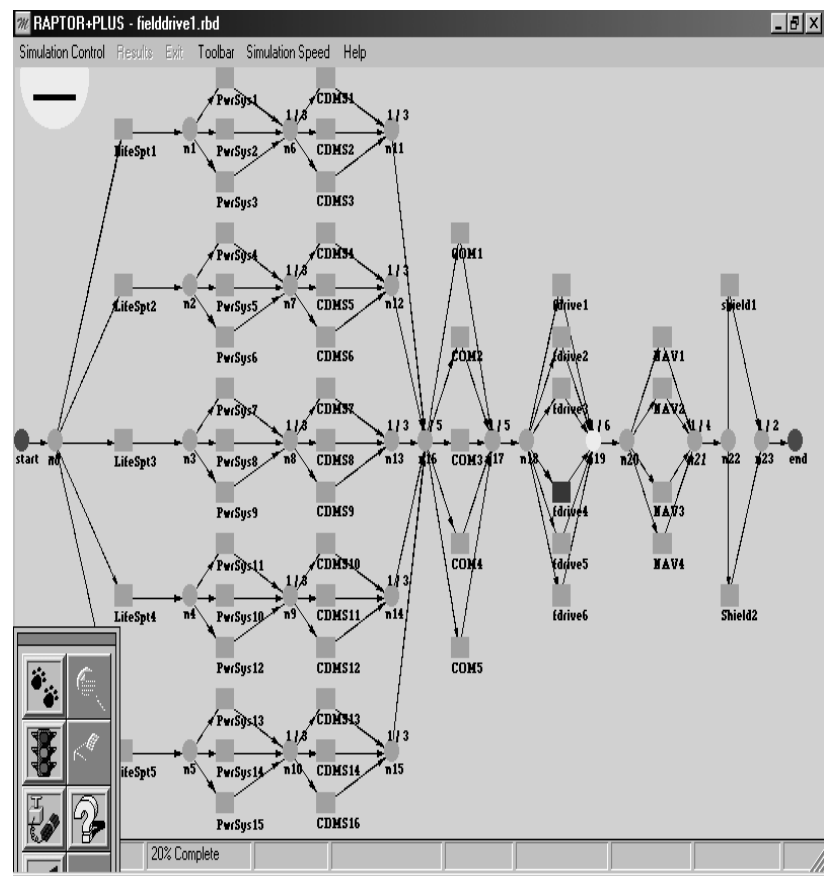

The third example of project analysis is the Success Tree Analysis of scientific research approaches. In this methodology, the research team defines a top-level success event for the project. In our example, this is defined as: "A Propulsion Physics Breakthrough." The intermediate events on the second level of the success tree would define events that may occur independently or in combination to bring about the top-level success event. Each of these intermediate events is further analyzed at level 3 of the success tree by using the appropriate logic gates. The Success Tree is developed as far down as the research team wishes to analyze or to the level of basic events. An example of the success tree is shown below in Fault Tree Logic text mode in a screen shot taken from SAPHIRE.

Figure 5: An Example Success Tree for the BPP Project

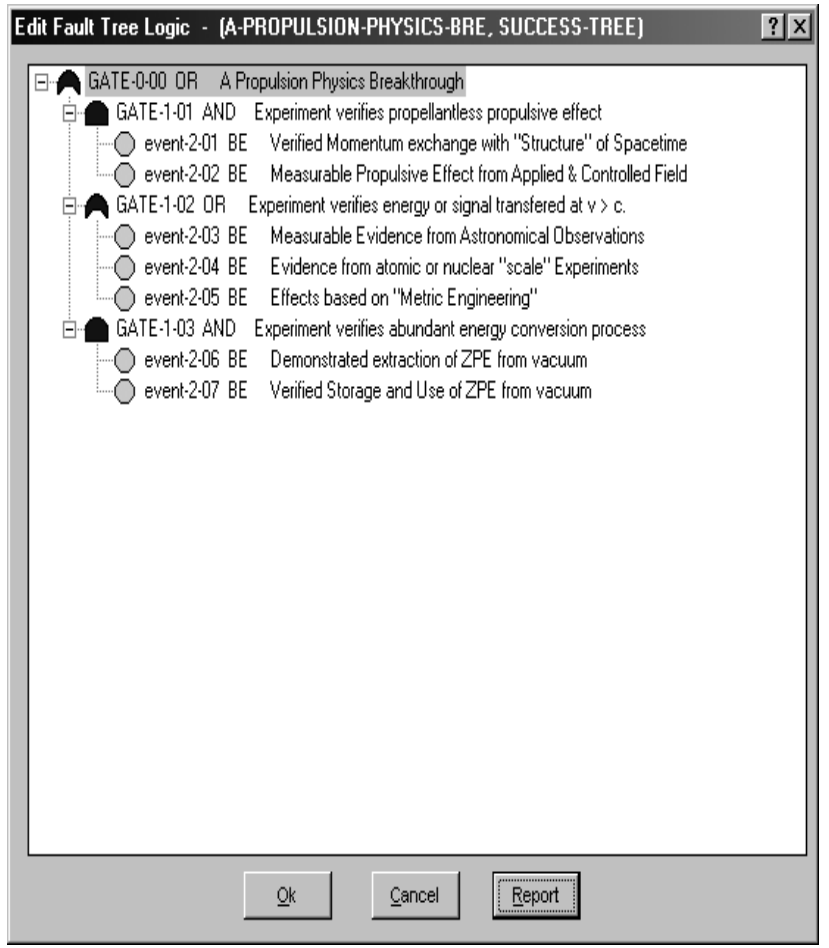

The Method of expert elicitation can be used to estimate the probability of success for basic success events at the lower tier of the success tree. The Fault tree tool can then be used to find the cut sets: the combination of basic success events that can cause the Top-Level success event. The minimal cut set can also be found: the smallest combination of basic success events that result in the Top-Level Success Event. The quantitative evaluations of a Success Tree consist of the determination of the Top-Event probability, the importance measures for the basic success events, sensitivity studies, and uncertainty analysis. (See Note 3) Uncertainty analysis calculates the variability in the success tree top event probability resulting from uncertainties in the basic event probabilities.

There are several important benefits from conducting the success tree analysis of the project. The Success Tree Analysis provides a visual, logic model of the basic and 
intermediate success events leading up to the top-level success event defined for the project. This draws attention to important basic success events and what must be done to enable these events. It also causes the consideration of human error, and its impact on preventing project success.

If a Success Tree is quantified, the basic success events that are the cause of project success can be prioritized according to their importance. It is expected that about $20 \%$ of the basic success events will contribute significantly to the top-Success Event probability.

\section{CONCLUSION}

There are three basic reasons to apply Risk Assessment to the Breakthrough Propulsion Physics Project, a project that is merely at stage of emerging science.

1. In relation to scientific experiments (experimental infrastructure and hardware) Risk analysis will address the real life potential hazards and system failure modes and how to avoid or mitigate them. This will improve our probability of success and provide safe experimentation.

2. It will improve the understanding of risks associated with hypothetical propulsion and propulsion vehicle concepts (in particular, field-drive concepts) in relation to possible interstellar missions.

3. Project Success Analysis or Success Tree Analysis has the capability to structure the possible scientific paths to success into a logical and visual model that captures the causal relationship between events. These models can be quantitatively evaluated to provide importance measures for specific basic success events. This process provides a tool to prioritize research efforts and creates a synergy with the emerging science.

\section{ACKNOWLEDGEMENT}

WE ARE PLEASED TO THANK the NASA management and engineering staff (especially NASA Headquarters, and JPL) who have taught us a great deal about Risk Assessment. We are also pleased to thank Kenneth E. Murphy of ARINC and Curtis L. Smith of the Idaho National Engineering \& Environmental Laboratory for their advice.

\section{NOTES}

Note (1)- "Fraction of science objectives achieved or gained" is a precise metric to enable quantification. However, many project scientists may typically think about this as "the amount of research progress" or "the amount of knowledge gained" which is a bit more subjective. Some scale for quantification may have to be developed.

Note (2)- In relation to a project like BPP at the maturity level of emerging science, event probabilities will not be available from actual performance data or data bases. In this situation, it is possible to estimate probabilities by using expert elicitation. This technique can provide fairly good estimates but care must be taken to be precise and repeatable on questions asked to the experts, to interview the actual experts, use both interviews and written forms for elicitation, and to pick a reasonable sample of experts for consultation.
Note (3) - SAPHIRE will solve the complemented fault tree logic ( the success tree), however it has not been optimized for such a solution. When large fault trees are involved the processing time may be significant. In addition, the "min cut upper bound" approximation that is used by SAPHIRE for minimal cut sets (to quantify their probability) sometimes leads to an overestimate of top-level success events when (a) basic events are not very independent from one another and (b) the cut set probabilities are large $(>0.1)$. Thus, the more careful approach would be to use the Min-Max quantification option in SAPHIRE. The Min-Max quantification option quantifies the current case study cut sets using the exact probability quantification algorithm.

\section{REFERENCES}

1. M.G.Millis, AD ASTRA, Pgs. 36-37, Jan. \& Feb. 1997.

2. E.Zampino, NASA Breakthrough Propulsion Physics Internal Report, "Critical Problems for Interstellar Propulsion Systems," June 1998

3. M. Alcubierre, "The warp-drive: hyper-fast travel within general relativity", Classical and Quantum Gravity, Vol.11,p. L73-L77, (1994)

4. D.C Cole, and H.E. Puthoff, "Extracting energy and heat from the vacuum”, Physical Review E, Vol. 48, No. 2, Aug. 1993, pp. 1562-1565.

5. M. Stamatelatos, J. Caraballo, W. Vesely, J. Dugan, J. Fragola, J. Minarick, J. Railsback, NASA Office of Safety and Mission Assurance, "Fault Tree Handbook with Aerospace Applications," April 30, 2002.

6. S.Cornford, "Tutorial on Defect Detection and Prevention (DDP): A Tool for Managing Risk", Engineering and Mission Assurance Directorate, NASA Jet Propulsion Laboratory, May 2000.

7. K.D. Russell, et al. "Systems Analysis Programs for Hands-on Reliability Evaluations (SAPHIRE)", Version 6.0 - System Overview Manual," NUREG/CR-6532, May 1999.

Edward J. Zampino

\section{BIOGRAPHIES}

NASA Glenn Research Center at Lewis Field

MS 50-4

21000 Brookpark Road

Cleveland, Ohio 44135 USA

e-mail: Edward.Zampino@grc.nasa.gov

Ed earned his MS degree in Physics from the Cleveland State University and worked as a Quality Assurance Engineer for the Technicare Division of Johnson \& Johnson. Ed became interested in Reliability when he had the opportunity to work with EIMAC Division of Varian, one of the world's largest producers of X-ray tubes. In 1987, he had the privilege to join NASA and has worked in Reliability engineering supporting many space flight projects. Ed has never left physics alone. He supports the NASA Breakthrough Propulsion Physics Project and is a private student of Dr. H. Yilmaz learning about relativistic theories of gravitation.

Marc G. Millis

NASA Glenn Research Center at Lewis Field

MS 86-2

21000 Brookpark Road

Cleveland, Ohio 44135 USA

e-mail: Marc.G.Millis@grc.nasa.gov

Mr. Millis has been with NASA's Glenn Research Center since 1982 after earning a degree in Physics from Georgia Tech. In addition to his more conventional engineering assignments that have included designing guidance displays for aerospace systems, he established the Breakthrough Propulsion Physics Project by developing collaborations with researchers across the nation. Mr. Millis managed this Project from 1996 through 2001, and has recently stepped down from Project Management to return to conducting research. Mr. Millis is also a graduate of the 1998 International Space University Summer Program. 